\title{
Isotropy group description of a phase transition in NANO3
}

\author{
W. Scott Stornetta \\ scott@mastertutors.org \\ Dorian M. Hatch \\ hatchd@byu.edu
}

Follow this and additional works at: https://scholarsarchive.byu.edu/facpub

Part of the Astrophysics and Astronomy Commons, and the Physics Commons

\section{Original Publication Citation}

Stornetta, Scott W. and Dorian M. Hatch."Isotropy group description of a phase transition in NaNO[sub 3]." The Journal of Chemical Physics 8 (1984): 5745-575.

\section{BYU ScholarsArchive Citation}

Stornetta, W. Scott and Hatch, Dorian M., "Isotropy group description of a phase transition in NANO3" (1984). Faculty Publications. 757.

https://scholarsarchive.byu.edu/facpub/757

This Peer-Reviewed Article is brought to you for free and open access by BYU ScholarsArchive. It has been accepted for inclusion in Faculty Publications by an authorized administrator of BYU ScholarsArchive. For more information, please contact ellen_amatangelo@byu.edu. 


\title{
Isotropy group description of a phase transition in $\mathrm{NaNO}_{3}$
}

\author{
W. Scott Stornetta and Dorian M. Hatch \\ Department of Physics and Astronomy, Brigham Young University, Provo, Utah 84602
}

(Received 16 December 1983; accepted 17 February 1984)

\begin{abstract}
Predictions of the possible post-transition symmetries of an $R \overline{3} c$ zone center continuous phase transition are made using recent extensions of the Landau theory. The Landau theory is summarized and direct group theoretical methods are applied to the $R \overline{3} c$ phase. General techniques for solving systems of nonlinear polynomial equations are reviewed and these techniques are applied to the minimization of the free energy $F$ for the aforementioned possible symmetries. The results of the direct methods and the minimization procedures are shown to be compatible. It is shown that the transition to $R 3 c$ in $\mathrm{NaNO}_{3}$ is consistent with the above results as well as with the observed atomic displacements.
\end{abstract}

\section{INTRODUCTION}

Landau theory, ${ }^{1}$ as extended by Birman, ${ }^{2}$ among others, predicts the symmetry which a crystal can have after a continuous (second order) phase transition. This prediction is based only on a knowledge of the symmetry of the crystal before the transition. This paper briefly explains the most recent version of this process, and applies the theory to the general consideration of a zone center transition of $R \overline{3} c$. The transition observed in $\mathrm{NaNO}_{3}$ is a continuous transition ${ }^{3}$ from $R \overline{3} c$ to $R 3 c$ proceding from a paraelectric to a ferroelectric phase. The transition occurs at room temperature and 45 $\mathrm{kbar}$ and is accompanied by $\mathrm{NO}_{3}^{-}$rotations as well as $\mathrm{Na}^{+}$ displacements. Since there is no change in primitive cell size the transition is a $\mathbf{k}=0$ soft mode. We show compatibility of the theory with the observed experimental evidence.

In Sec. II we review the original Landau theory, making a connection with the more recent developments in the theory, viz., the subduction and chain subduction criteria, and the related concept of an isotropy group. The latter direct group theoretical methods including the Landau and the Lifshitz conditions are then applied to $R \overline{3} c$ for the zone center yielding six possible post-transition subgroups.

In Sec. III we consider the additional restriction of minimizing the free energy $F$. Thus generally a subset of the groups obtained by direct methods correspond to a minimum of $F$. We review the method of Jarić $c^{4}$ for solving systems of nonlinear polynomial equations, exploiting the invariance of these equations under relevant symmetry groups. These methods use projection operators to partition solutions of $\nabla F=0$ into sets according to the symmetry of the vector order parameter, and thereby reduce the degree of the relevant equations. We apply the techniques for the free energy $F$ expanded to fourth order (simple continuous transition).

In Sec. IV using a lattice atomic model, we then indicate the agreement between the predicted order parameter symmetry and experiment for the $\mathrm{NaNO}_{3}$ transition including the determination of the distortion from the average position of the atoms during the transition.

\section{LANDAU THEORY AND ISOTROPY GROUPS}

The symmetry of a crystal can be defined in terms of a density function, $\rho(x, y, z)$, after Landau. ${ }^{1}$ The symmetry group of a crystal is then the set of rotations, reflections, translations, etc., that leave $\rho$ unchanged. Thus, letting $r$ represent an arbitrary transformation, the symmetry group $R_{0}$ is the set

$$
R_{0} \equiv\{r: 0, \rho(x, y, z)=\rho(x, y, z)\} \text {. }
$$

For the structure of $\mathrm{NaNO}_{3}$, which is of interest here, there are 12 coset representatives which may be selected (cosets with respect to translations) in the space group (see Fig. 1):

$E$ : The identity element. All atoms and coordinates are unchanged. $C_{3}{ }^{ \pm}$: A rotation about the $z$ axis by $120^{\circ}$, clockwise $(-)$ or counterclockwise $(+) . C_{21}^{\prime}$ : A $180^{\circ}$ rotation about the axis along a line from 5 to $3 . C_{22}^{\prime}$ : Same as $C_{21}^{\prime}$ only along a line from 7 to $3 . C_{23}^{\prime}$ : Same as $C_{21}^{\prime}$ only along a line from 6 to 3. $I$ : Inversion through 2. $S_{6}^{ \pm}: I C_{3}^{ \pm}$as defined above. $\sigma_{d 1}, \sigma_{d 2}, \sigma_{d 3}$ : Reflection through a plane containing the $z$ axis and perpendicular to the $C_{21}^{\prime}, C_{22}^{\prime}$, and $C_{23}^{\prime}$ axes, respectively, followed by a glide of $1 / 2$ of a unit cell.

This collection of 12 cosets together with trigonal translations, is $R \overline{3} c$ in the international notation, ${ }^{5}$ and its related point group $(G / T)$ is $D_{3 d}$. The origin of $R \overline{3} c$ labeling is at the $\overline{3}$ center, i.e., at the $\mathrm{Na}$ atom in $\mathrm{NaNO}_{3}$.

For a quite arbitrary density function $\rho$ it is well known from group theory that the function can be expanded in terms of symmetry functions with respect to $R \overline{3} c$, i.e.,

$$
\rho=\rho_{0}+\sum_{m}^{\prime} \sum_{i} \eta_{i}^{m} \Psi_{i}^{m} \equiv \rho_{0}+\Delta \rho
$$

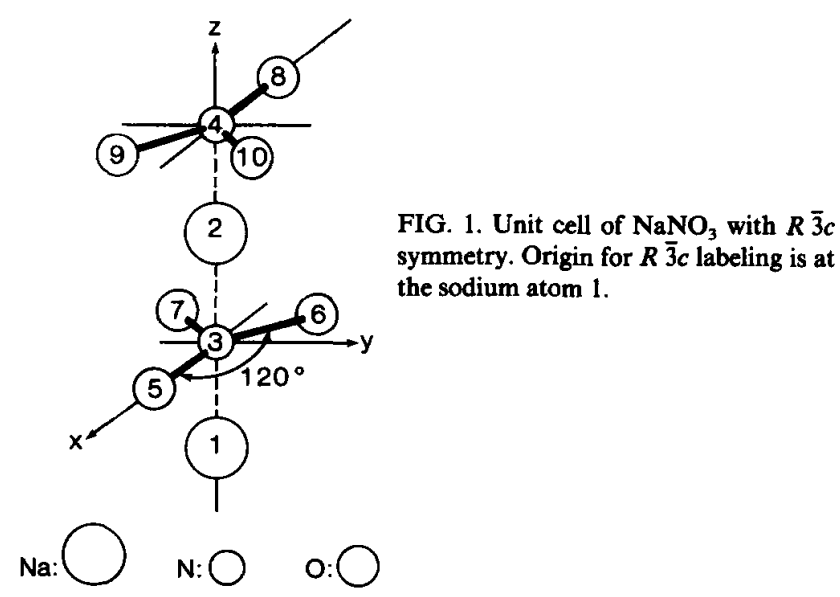


where the prime on the first summation indicates that the identity representation is not included in the sum but is contained in $\rho_{0}$. Here $m$ labels the representation and $i$ labels the $i$ th basis function of the $m$ th representation. Thus for a phase transition, we take $\rho_{0}$ to be the pretransition density with symmetry $R_{0}(R \overline{3} c)$ and $\rho$ to be the post-transition density. Because $\rho$ contains additional components not invariant un$\operatorname{der} R_{0}$, the symmetry is lowered to a subgroup.

The free energy of the crystal must be at a local minimum for any particular phase of the crystal to be stable. In general, $F$ is a functional of the density function, pressure, temperature, and any external fields. But from Eq. (2), $\rho$ can be expressed in terms of the $\Psi_{i}^{m}$, and therefore an explicit dependence of $F$ on the $\eta_{i}^{m}$ results. It is a characteristic of a continuous phase transition that the coefficients $\eta_{i}^{m}$ vary continuously from zero as one passes through the transition point. This condition allows us to write the free energy in a series expansion of the $\eta_{i}^{m}$ about the transition point. The expansion must satisfy the requirement that it be invariant under an arbitrary symmetry transformation of the space group. To guarantee this invariance the most general form must be combinations of the $\eta_{i}^{m}$ each of which are invariant under transformations of the space group, i.e., transformations of the form $g \eta_{i}=D_{i j} \eta_{j}$. We assume a single irrep induces the transition. At fourth order only two such invariants ${ }^{6}$ come into play and we exclude representations with third order invariants. Thus $\rho$ has the form

$$
\rho=\rho_{0}+\sum_{i} \eta_{i} \Psi_{i},
$$

while

$$
F=F_{0}+A \sum_{I} \eta_{i}^{2}+\sum_{\alpha} B_{\alpha} f_{\alpha}^{4}\left(\eta_{i}\right)
$$

Here the $f_{\alpha}^{4}\left(\eta_{i}\right)$ are fourth order homogenous invariant functions of $\eta_{i}$.

To determine possible symmetries of the post-transition phase, we must find the set of elements which leave $\rho$ invariant. Of course, $\rho_{0}$ is invariant under all elements $R_{0}$. Also, it is clear that $\Sigma \eta_{i} \Psi_{i}$ is invariant under all elements whose corresponding matrix in the irrep $\Gamma$ is the identity matrix. These elements form a subgroup, the kernel of $\Gamma$. The invariance of $\rho$ under the elements of the kernel holds for arbitrary values of $\eta_{i}$. However, for a particular choice of $\eta$, say $\eta^{\prime}$, the sum $\Sigma \eta_{i}^{\prime} \Psi_{i}$ may be invariant under additional elements, which form a subgroup containing the kernel. The largest group leaving the sum invariant will be called an isotropy group of $R_{0}$ in $\Gamma$. Because the isotropy groups are the only possible symmetries of $\Delta \rho$, the low-symmetry phase must be an isotropy group corresponding to an irrep of the highsymmetry phase.

Birman ${ }^{7} \mathrm{saw}$ in the classical formalism of Landau a necessary group theoretical condition on the lower symmetry group. If we let $\chi(r)$ be the character of the $\Gamma(r)$ matrix of a reducible representation corresponding to the transformation $r$, and let $\chi^{p}(r)$ be the character of the $\Gamma^{p}(r)$ matrix corresponding to $r$ of the $p$ th irrep then the number of times $\Gamma^{p}$ enters into the reducible representation is given by $i$, where

$$
i=\frac{1}{|R|} \sum_{r \in R} \chi^{p}(r) \chi(r)
$$

The statement that $R^{\prime}$ be an isotropy group implies that the identity irrep of $R^{\prime}$ be contained (subduced) in the irrep $\Gamma^{m}$ of $R_{0}$ (subduction criterion ${ }^{5}$ ), i.e.,

$$
i\left(R^{\prime}\right)=\frac{1}{\left|R^{\prime}\right|} \sum_{r \in R^{\prime}} \chi^{m}(r) \neq 0 .
$$

It is worth noting that the kernel is an isotropy group such that $i\left(R^{\prime}\right)=\operatorname{dim} \Gamma^{m}$. Thus, for a continuous phase transition from a high-symmetry to a low-symmetry phase, the only possible post-transition symmetries are those for which $i\left(R^{\prime}\right)$ is not equal to zero.

The character of a general space group element is

$$
\chi(g)=\sum_{\sigma} e^{-\tau \mathbf{k}_{\sigma} \cdot \mathbf{t}} \chi\left(\left\{r_{\sigma} \mid \tau_{\sigma}\right\}^{-1} g\left\{r_{\sigma} \mid \tau_{\sigma}\right\}\right)
$$

where the $\sigma$ sum is over the arms of the star. For the restriction to the $k=0$ point only the representations of the point groups need to be considered and only one arm occurs. Bradley and Cracknel, ${ }^{8}$ give the irreps of $D_{3 d}\left(G_{12}^{3}\right)$ as $A_{1 \mathrm{~g}}\left(R_{1}\right)$, $A_{2 g}\left(R_{2}\right), A_{1 u}\left(R_{3}\right), A_{2 u}\left(R_{4}\right), E_{g}\left(R_{5}\right)$, and $E_{u}\left(R_{6}\right)$. In the case of one-dimensional irreps, the kernel is the only isotropy group. As an example, the character table for the $A_{2 u}$ $\left(R_{4}\right)$ irrep of $D_{3 d}$ is

\begin{tabular}{cccccc}
$E$ & $2 C_{3}$ & $3 C_{2}^{\prime}$ & $i$ & $2 S_{6}$ & $3 \sigma_{d}$ \\
\hline 1 & 1 & -1 & -1 & -1 & 1
\end{tabular}.

By noting which elements correspond to identity matrices for the irrep $A_{2 u}$ the elements of the kernel for $A_{2 u}$ correspond to $C_{3 v}$ and are thus

$$
\begin{aligned}
& \{E \mid 000\}, \quad\left\{C_{3} \mid 000\right\}, \quad\left\{C_{3}^{-} \mid 000\right\}, \quad\left\{\sigma_{d 1} \mid \frac{1}{2} \frac{1}{2} \frac{1}{2}\right\}, \\
& \left\{\sigma_{d 2} \mid \frac{1}{2} \frac{1}{2} \frac{1}{2}\right\}, \quad\left\{\sigma_{d 3} \mid \frac{1}{2} \frac{1}{2} \frac{1}{2}\right\}
\end{aligned}
$$

together with all translations. This space group is the group $C_{3 v}^{6}$. We have used the notation of Refs. 4 and 8 for the labeling of the symmetry elements above.

To find the isotropy groups of the two-dimensional irreps we use the formula for the subduction frequency. For example, in the case of the $E_{g}$ irrep, the character table is

\begin{tabular}{cccccc}
$E$ & $2 C_{3}$ & $3 C_{2}^{\prime}$ & $i$ & $2 S_{6}$ & $3 \sigma_{d}$ \\
\hline 2 & -1 & 0 & 2 & -1 & 0
\end{tabular}.

Applying Eq. (8) to this irrep and using the isomorphism between the isogonal point group and the coset representatives (remember $\mathbf{k}=0$ )

$$
i\left(C_{i}\right)=\frac{1}{\left|C_{i}\right|}\{\chi(E)+\chi(i)\}=\frac{1}{2}\{2+2\}=2,
$$

as $C_{i}$ is the kernel of the $E_{g}$ irrep of $D_{3 d}$. By evaluating the subduction frequency of all subgroups of $D_{3 d}$ we determine the invariance subgroups of the two-dimensional irreps of $D_{3 d}$ which have nonzero subduction frequency for each irrep.

An additional restriction on the possible resultant groups of a continuous phase transition is Birman's chain subduction criterion. ${ }^{9}$ This contains the physical statement that the symmetry group must be the largest subgroup which leaves the subspace invariant, i.e., an isotropy group as defined earlier. It takes the following form: Suppose two subgroups, $R_{1}$ and $R_{2}$, satisfy

$$
R_{1} \subset R_{2},
$$




$$
i\left(R_{1}\right)=i\left(R_{2}\right) \text {. }
$$
metry.

Then $R_{1}$ is ruled out as a possible post-transition sym-

Thus, using the preceeding group theoretical considerations alone, we reduce the number of possible point groups to nine, namely: $D_{3 d}, C_{3 i}, C_{i}, C_{2 h}, D_{3}, C_{3}, C_{1 h}, C_{2}$, and $C_{1}$.

The classical Landau formulation also contains two additional group theoretical conditions. ${ }^{1}$ The first condition derives from the condition that the free energy be stable at the origin, i.e., $\boldsymbol{\eta}=0$. This requires that the third order term in the expansion be absent. Thus the symmetrized triple Kronecker product of the representation $\Gamma^{m}$ must not contain the identity representation of $R_{0}$, i.e.,

$$
\left[(\Gamma)^{3} \mid \Gamma^{+}\left(R_{0}\right)\right]=0 .
$$

The second condition comes from the requirement that the less symmetric phase be stable relative to nonspatially periodic perturbations. This condition requires that the antisymmetrized double Kronecker product of $\Gamma$ not contain the representation of a polar vector of $R_{0}$, i.e.,

$$
\left[\{\Gamma\}^{2} \mid V\left(R_{0}\right)\right]=0
$$

Considering the above six representations the $A_{1 g}$ and $E_{g}$ representations must be discarded as they do not satisfy the Lifshitz condition. We thus obtain Table I. The above result is consistent with those obtained in Ref. 10. For the transition of interest in $\mathrm{NaNO}_{3}$ we see that direct group methods allow the isotropy group $R 3 c$ corresponding to ir$\operatorname{rep} A_{2 u}$.

\section{USE OF SYMMETRY IN SOLVING $\nabla F=0$}

Landau theory places the additional constraint that a phase transition corresponds to a minimum of the free energy at $\eta_{i} \neq 0$. (Due to the transformation properties of the $\eta$, viz., $\eta_{i}^{\prime}=D_{i j} \eta_{i}$, we will consider $\eta_{i}$ as a vector representation and denote it as $\eta$.) We thus seek solutions to the equation

$$
\nabla F \equiv \mathbf{f}(\boldsymbol{\eta})=0,
$$

and in particular wish to determine if $R 3 c$ corresponds to a minimum. We will discuss the problem in general terms initially. It is easy to verify that $f$ has the form

$$
f_{i}(\eta)=\sum_{\alpha} A_{\alpha}(\eta) \eta_{i}^{2 \alpha+1},
$$

with $A_{\alpha}(\eta)$ an invariant function of $\eta$. Because of the large

TABLE I. Isotropy subgroups corresponding to $\mathbf{k}=0$.

\begin{tabular}{lcc}
\hline \hline Irrep & Subgroup & Subduction frequency \\
\hline$A_{18}{ }^{a}$ & $R \overline{3} c\left(D_{3 d}^{6}\right)$ & 1 \\
$A_{28}$ & $R \overline{3}\left(C_{3 i}^{2}\right)$ & 1 \\
$A_{1 u}$ & $R_{32}\left(D_{3}^{7}\right)$ & 1 \\
$A_{2 u}$ & $R \overline{3} c\left(C_{3 v}^{6}\right)$ & 1 \\
$E_{g}$ & $P \overline{1}\left(C_{i}^{1}\right)$ & 2 \\
& $C 2 / c\left(C_{2 h}^{6}\right)$ & 1 \\
$E_{u}$ & $P 1\left(C_{t}^{1}\right)$ & 2 \\
& $C c\left(C_{S}^{4}\right), C_{2}\left(C_{2}^{3}\right)$ & 1
\end{tabular}

"Representations which do not satisfy the Lifshitz condition. number of solutions for the general situation as well as the resulting symmetry of the system we would benefit from a systematic approach to finding solutions. A proposed approach was given by Jaric, ${ }^{3}$ and is briefly reviewed and applied in this section. Jaric's method exploits the fact that the solutions to Eq. (10) can be selected according to the isotropy groups of $\boldsymbol{R}_{0}$.

To begin with, because $F$ was constructed to be invariant under $R_{0}$, it follows that:

$$
\mathbf{f}(\boldsymbol{\eta})=r f(\eta)
$$

where both $\eta$ and $f$ are $n$-dimensional vectors. We now define a hyperplane fix $R$ as the set of all vectors which are left invariant by the isotropy group $R$. Also we can speak of hyperplanes fix $R$ for both $\mathrm{f}$ and $\eta$. Now let $\eta \epsilon$ fix $R$. From Eq. (12) it follows that $r(\eta)=f(\eta)$, because $r$ leaves $\eta$ invariant. Therefore, $\eta \in$ fix $R$ implies $\mathrm{f}(\eta) \in$ fix $R$.

To exploit the symmetry of $f(\eta)$ and $\eta$, we construct the projection operator

$$
P(R)=\frac{1}{|R|} \sum_{r \in R} 0_{r},
$$

which projects an arbitrary vector $\eta$ (or $f$ ) onto the hyperplane fix $R$. We expect $\eta$ to exhibit the symmetry of the posttransition phase. Therefore, $\eta$ will lie in a fix $R$, where $R$ is one of the possible isotropy groups, e.g., those obtained in Sec. II. Thus, we wish to partition solutions to Eq. (10) according to the hyperplane in which $\eta$ lies, or in other words, according to the symmetry of $\boldsymbol{\eta}$. The equation

$$
[1-P(R)] \eta=0
$$

restricts us to those values of $\eta$ lying in fix $R$. For this case $\mathrm{f}(\boldsymbol{\eta}) \epsilon$ fix $R$ and we can replace Eq. (10) with

$$
P(R) \mathbf{f}(\boldsymbol{\eta})=0 \text {. }
$$

From Table I, we see that some isotropy groups are subgroups of others but with different subduction frequencies. In fact, a particular nesting of isotropy groups, allows us to exploit the partitioning of solutions in a way that reduces the difficulty of the equations. Suppose two isotropy groups, $R$ and $R_{k}$, are related as follows:

$$
R \subset R_{k}
$$

and

$$
i\left(R_{k}\right)=i(R)-1
$$

Note that from Eq. (16) it follows immediately that fix $R$ $\supset$ fix $R_{k}$.

Consider the operator $\left[P(R)-P\left(R_{k}\right)\right]$. On an arbitrary vector, this operator yields a vector lying in fix $R$, but with no component in fix $R_{k}$. Also, because the dimension of fix $R_{k}$ is only one less than that of fix $R$, any two vectors lying in fix $R$ but having no component in fix $R_{k}$ must be collinear. Therefore, we can write

$$
\left[P(R)-P\left(R_{k}\right)\right] \mathbf{f}(\boldsymbol{\eta}) \equiv g_{k}(\boldsymbol{\eta})\left[P(R)-P\left(R_{k}\right)\right] \boldsymbol{\eta},
$$

where $\left[P(R)-P\left(R_{k}\right)\right] f(\eta)$ and $\left[P(R)-P\left(R_{k}\right)\right] \eta$ are twocollinear vectors that differ only by a factor $g_{k}(\eta)$, and where Eq. (17) defines $g_{k}(\eta)$. The condition

$$
\left[P(R)-P\left(R_{k}\right)\right] \mathrm{f}(\boldsymbol{\eta})=0,
$$




$$
g_{k}(\eta)=0 .
$$

This yields all solutions to Eq. (10) for $\eta$ in fix $R$ and yet which have no component lying in fix $R_{k}$. Notice that Eq. (19) is of $1^{\circ}$ less than Eq. (10), and hence easier to solve.

To summarize the general technique to this point, in seeking solutions to a set of nonlinear equations, we restrict our search to those solutions with a particular isotropy group symmetry, obtain a relevant equation from which we can remove a known factor and thus eliminate spurious solutions, and as a result reduce our equations by $1^{\circ}$.

As Jarić points out, in general there could be several isotropy groups, call them $R_{1}, R_{2}, \ldots, R_{m}$ which satisfy Eqs. (16a) and (16b), and each such isotropy group yields a distinct function $g_{1}, g_{2}, \ldots, g_{m}$. In fact, typically the number of isotropy groups $(m)$ is larger than the dimension $i(r)$ of fix $R$. Because the normals to the hyperplanes fix $\left(R_{j}\right)$ span fix $R$, the solutions to $g_{k}=0, k=1, \ldots, i(r)$, contain all the solutions in fix $R$ and the additional $g_{k}$ are not linearly independent solutions. To handle this case, additional considerations can be introduced which for our $\mathbf{k}=0$ case are not necessary.

Thus let us apply the preceeding theory to the $\mathbf{k}=0$ representations of $R \overline{3} c$. One example of the method will be shown explicitly.

Choose $R$ to be $C_{i}$, corresponding to the $E_{g}$ irrep. $R_{k}$ is then $C_{2 h}$. For a two-dimensional irrep we represent the vector $\eta$ with two components, $\eta_{1}$ and $\eta_{2}$. Then the free energy to fourth order is

$$
F=F_{0}+A\left(\eta_{1}^{2}+\eta_{2}^{2}\right)+C\left(\eta_{1}^{2}+\eta_{2}^{2}\right)^{2}
$$

and

$$
\mathbf{f}(\boldsymbol{\eta})=\left[2 A+4 C\left(\eta_{1}^{2}+\eta_{2}^{2}\right)\right]\left(\begin{array}{l}
\eta_{1} \\
\eta_{2}
\end{array}\right),
$$

which is seen to have the general form of Eq. (11). To separate all solutions with $C_{i}$ symmetry we construct

$$
P\left(C_{i}\right)=1 / 2\left[\left(\begin{array}{ll}
1 & 0 \\
0 & 1
\end{array}\right)+\left(\begin{array}{ll}
1 & 0 \\
0 & 1
\end{array}\right)\right]=\left(\begin{array}{ll}
1 & 0 \\
0 & 1
\end{array}\right) .
$$

Equation (14) becomes

$$
\left[\left(\begin{array}{ll}
1 & 0 \\
0 & 1
\end{array}\right)-\left(\begin{array}{ll}
1 & 0 \\
0 & 1
\end{array}\right)\right]\left(\begin{array}{l}
\eta_{1} \\
\eta_{2}
\end{array}\right)=0
$$

which admits all values of $\left(\begin{array}{l}\eta_{1} \\ \eta_{2}\end{array}\right)$. We now determine $P\left(C_{2 h}\right)$ :

$$
\begin{aligned}
P\left(C_{2 h}\right)= & \frac{1}{4}\left[\left(\begin{array}{ll}
1 & 0 \\
0 & 1
\end{array}\right)+\left(\begin{array}{ll}
1 & 0 \\
0 & 1
\end{array}\right)+\left(\begin{array}{rr}
1 & 0 \\
0 & -1
\end{array}\right)\right. \\
& \left.+\left(\begin{array}{rr}
1 & 0 \\
0 & -1
\end{array}\right)\right]=\left(\begin{array}{ll}
1 & 0 \\
0 & 0
\end{array}\right)
\end{aligned}
$$

and construct

$$
\left[P\left(C_{i}\right)-P\left(C_{2 h}\right)\right]=\left(\begin{array}{ll}
0 & 0 \\
0 & 1
\end{array}\right)
$$

Thus a vector $\eta$ contained in fix $C_{i}$ but not in fix $C_{2 h}$ will have only an $\eta_{2}$ component. Equation (17) becomes

$\left(\begin{array}{ll}0 & 0 \\ 0 & 1\end{array}\right)\left\{\left[2 A+4 C\left(\eta_{1}^{2}+\eta_{2}^{2}\right)\right]\left(\begin{array}{l}\eta_{1} \\ \eta_{2}\end{array}\right)=g_{C_{2 h}}\left[\left(\begin{array}{ll}0 & 0 \\ 0 & 1\end{array}\right)\left(\begin{array}{l}\eta_{1} \\ \eta_{2}\end{array}\right)\right]\right.$.

Solving for $g_{C_{2 \hbar}}$, while requiring $\left[P\left(C_{i}\right)-P\left(C_{2 h}\right)\right] \eta \neq 0$ yields

$$
g_{C_{2 h}}=2 A+4 C \eta_{2}^{2}=0 \text {. }
$$

Therefore,

$$
\eta_{2}= \pm\left[\frac{-A}{2(C)}\right]^{1 / 2}
$$

is a solution corresponding to symmetry $C_{i}$ but not the higher symmetry $C_{2 h}$. Minima for the other isotropy groups can be obtained in a similar fashion. It is easily seen that all isotropy groups obtained in Sec. II correspond to minima of the corresponding free energy to fourth order in $\eta$. Our interest is mainly in $A_{2 u}$ and $R 3 c$ and one sees that $R 3 c$ does correspond to a minimum of the generalized free energy $F=F_{0}+A \eta_{1}^{2}+B \eta_{1}^{4}$ with $\eta_{1}= \pm \sqrt{(-A / 2 B)}$ and $A<0$, $B>0$.

\section{LATTICE DESCRIPTION}

Obtaining a simple form for the density function yields additional insight into the movement of atoms during the phase transition. We will assume an average equilibrium position for each atom: $\mathbf{r}=\left(\mathbf{r}_{1}, \mathbf{r}_{2}, \ldots \mathbf{r}_{n}\right)$. Also displacements from equilibrium $u_{i}$ are assumed to be small. Then $\rho(\mathbf{x}-\mathbf{r}-\mathbf{u}) \approx \rho(\mathbf{x}-\mathbf{r})-\nabla \rho \cdot \mathbf{u} \equiv \rho_{0}-\nabla \rho \cdot \mathbf{u}$. Consistent with the Landau philosophy the displacements from equilibrium must correspond to the single irrep of the transition.

Perhaps the simplest coordinate system for describing the displacements from equilibrium $\mathbf{u}$ is a set of Cartesian coordinates, one set being attached to each atom. Then the arbitrary displacements can be written $\mathbf{u}_{1}=\mathbf{u}_{1 x} \mathbf{i}_{1}+u_{1_{y}}$ $\mathbf{j}_{1}+u_{1 z} k_{1}$ similarly for each $\mathbf{u}_{i}$ where $i$ labels an atom of the unit cell.

We could equivalently write a column matrix which represents a vector in a vector space spanned by the basis functions $i_{1}, j_{1}, k_{1}, \ldots, k_{n}$. The fact that any arbitrary displacement $\mathbf{u}$ can be written as a linear combination of basis functions allows us to know the effect of $0_{r}$ on a general displacement $u$ simply by knowing its effect on each of the basis functions $i_{1}, \mathbf{j}_{1}, \mathbf{k}_{1}, \ldots, \mathbf{k}_{n}$. This then allows us to represent each operator by a matrix $\Gamma_{i j}(r)$. Thus, if

$$
0_{r} \mathbf{r}=0_{r}\left(\mathbf{r}_{0}+\mathbf{u}\right)=0_{r} \mathbf{r}_{0}+0_{r} \mathbf{u}=\mathbf{r}_{0}+\mathbf{u}^{\prime},
$$

then

$$
\sum_{j} \Gamma_{i j} u_{j}=u_{i}^{\prime} .
$$

The ten-atom set of $\mathrm{NaNO}_{3}$ has a set of $30 \times 30$ dimensional matrices to represent transformations on the unit cell. From Fig. 1 and the description of the symmetry tranformations the matrices corresponding to the various elements of $R_{0}$ are easily determined. The matrices form a reducible representation $\Gamma$ of $R \overline{3} c$ and therefore, we can reduce the representation in terms of the irreducible representations of $\mathbf{k}=0$. Recall that the number of times an irrep enters a given reducible representation is given by Eq. (7). The results of the calculation are $A_{1 g}: 1, A_{2 g}: 3, E_{g}: 4, A_{1 u}: 2, A_{2 u}: 4, E_{u}: 6$. Remembering that each $E_{g}$ or $E_{u}$ irrep has two basis functions associated with it, we can see that the above reduction agrees with the dimension of the original matrix.

In changing the original $30 \times 30$ matrix to a set of irreducible component representations we also must change the basis functions. These new functions must transform as basis 
functions of the respective irreps. A systematic method for obtaining these basis function makes use of projection operators.

The formula for the $i$ th basis function of the $m$ th irrep is

$$
P_{i}^{m}(R) \Psi=\frac{\operatorname{dim} I^{m}}{|R|} \sum_{r \in R} \Gamma_{i j}^{m}(r) 0, \Psi
$$

where $\psi$ is an arbitrary vector and $j$ is fixed. Here we represent $\Psi$ by its components in the 30-dimensional Cartesian space and the operator $0_{r}$ is the corresponding matrix in the 30-dimensional space. An example calculation, using the projection operator, is sketched below for $A_{2 u}$.

$$
\begin{aligned}
P_{1}^{A} 2 u\left(D_{3 d}\right) \Psi= & \frac{1}{\left|D_{3 d}\right|}\left\{\Gamma_{11}^{A_{2 u}}(E) E \Psi+\Gamma_{11}^{A_{2 u}\left(C_{3}{ }^{+}\right) C_{3}^{+} \Psi}\right. \\
& \left.+\cdots+\Gamma_{11}^{A_{2 u}}\left(\sigma_{d 3}\right) \sigma_{d 3} \Psi\right\} .
\end{aligned}
$$

Because the operators $0_{r}$ are linear we can separate out the sum of matrices:

$$
P_{1}^{A} 2 u\left(D_{3 d}\right) \Psi=\frac{1}{\left|D_{3 d}\right|}\left[\sum_{r} \Gamma_{11}^{A_{2 u}}(r) 0_{r}\right] \Psi .
$$

The sum of matrices, with the appropriate coefficients, is shown in the Appendix. If we choose $y_{5}$ for our initial $\Psi$, then

$$
\begin{aligned}
& A_{1}^{A_{2 u}}\left(D_{3 d}\right) y_{5}= 2 y_{5}+2 \gamma x_{6}-y_{6}-2 \gamma x_{7}-y_{7}+2 y_{8} \\
&+2 \gamma x_{9}-y_{9}-2 \gamma x_{10}-y_{10}, \\
& \gamma=(3 / 2)^{1 / 2},
\end{aligned}
$$

which represents an alternating rotation of the nitrate groups. A similar procedure choosing $z_{2}$ as an initial $\Psi$ yields $z_{1}+z_{2}$ corresponding to a displacement of the $\mathrm{Na}$ atoms along $k$.

In determining basis functions for the irreps we find, e.g., that one possible basis function for $A_{2 u}$ is $z_{1}+z_{2}+\cdots+z^{10}$, which is simply a translation of the entire unit cell. Such a vector says nothing about the internal symmetry of the crystal. We eliminate this function and corresponding ones for translation in the $x-y$ plane, and we are left with 27 basis functions, sufficient to describe any relative displacements of atoms within the crystal. The remaining irreps then enter as follows: $A_{1 g}: 1, A_{2 g}: 3, E_{g}: 2, A_{1 u}: 3, A_{2 u}$ : 4, $E_{u}: 5$.

In general, for the displacement vector to transform as a given irrep it can be a linear combination of the vector basis functions. This is in fact the case for the $\mathbf{4 5} \mathrm{kbar}$ transition in $\mathrm{NaNO}_{3}$. Barnett ${ }^{3}$ reported that this transition involves the rotation of alternating nitrate groups by $15^{\circ}$ around the $z$ axis, coupled with a translation of the sodium atoms along the $z$ axis. Thus a linear combination of the two basis functions indicated above does in fact correspond to the experimental displacements and it transforms according to the $A_{2 u}$ irrep. This irrep is the same one which obtains the lower symmetry phase $R 3 c$. The group theoretical description then does yield a compatible description of the experimental results for the $\mathrm{NaNO}_{3}$ transition.

\section{CONCLUSIONS}

We have shown that direct group theoretical methods appropriate to the description of continuous phase transitions yield six possible lower symmetry phases corresponding to the $\mathrm{k}=0$ irreps of $R \overline{3} c$. We used general techniques of solving polynomial equations to find solutions corresponding to the extrema of the free energies to fourth order for these six phases. Although the full use of these techniques is not needed here it is shown that all phases correspond to minima. We have also shown that the $A_{2 u}$ representation drives the transition $R \overline{3} c$ to $R 3 c$ in $\mathrm{NaNO}_{3}$ and yields the appropriate equilibrium distortions of the atoms as the system goes through the transition.

\section{ACKNOWLEDGEMENTS}

The authors are grateful for helpful discussions with Professor J. D. Barnett, Professor Harold T. Stokes, Professor Daniel L. Decker, and Professor R. Vencil Skarda as well as Jeffery W. Felix. 


\section{APPENDIX}

The form for the projection operator $P_{1} A_{2 u}\left(D_{3 d}\right)$ of Eq. (17). It is a $10 \times 10$

block matr1x where each entry is a $3 \times 3$ matrix of the form listed below.

\begin{tabular}{|c|c|c|c|c|c|c|c|c|c|}
\hline $\begin{array}{c}\mathrm{E}+\mathrm{C}_{3} \\
+\mathrm{c}_{3}^{-}-1 \\
-\mathrm{s}_{6}^{+}-\mathrm{s}_{6}^{-}\end{array}$ & $\begin{array}{c}\sigma_{1}+\sigma_{2} \\
+\sigma_{3}-C_{21} \\
-C_{22}-C_{23}\end{array}$ & & & & & & & & \\
\hline $\begin{array}{c}\sigma_{1}+\sigma_{2} \\
+\sigma_{3}-c_{21} \\
-c_{22}-c_{23}\end{array}$ & $\begin{array}{c}E_{+}+C_{3}^{+} \\
+C_{3}^{-}-i \\
-s_{6}^{+}-s_{6}^{-}\end{array}$ & & & & & & & & \\
\hline & & $\begin{array}{c}E+C_{3}^{+} \\
+c_{3}^{-}-c_{21} \\
-c_{22}-c_{23}\end{array}$ & $\begin{array}{c}\sigma_{1}+\sigma_{2} \\
+\sigma_{3}-1 \\
-s_{6}^{+}-s_{6}^{-}\end{array}$ & & & & & & \\
\hline & & $\begin{array}{c}\sigma_{1}+\sigma_{2} \\
+\sigma_{3}^{-i} \\
-s_{6}^{+}-s_{6}^{-}\end{array}$ & $\begin{array}{c}E+C_{3}^{+} \\
+C_{3}^{-}-C_{21} \\
-C_{22}-C_{23}\end{array}$ & & & & & & \\
\hline & & & & $E-c_{21}$ & $c_{3}^{+}-c_{22}$ & $c_{3}^{-}-c_{23}$ & $\sigma_{1}-\mathbf{i}$ & $\sigma_{2}-S_{6}^{-}$ & $\sigma_{3}-s_{6}^{+}$ \\
\hline & & & & $c_{3}^{-}-c_{22}$ & $\mathrm{E}-\mathrm{C}_{23}$ & $c_{3}^{+}-c_{21}$ & $\sigma_{2}-s_{6}^{+}$ & $\sigma_{3}-1$ & $\sigma_{1}-\mathrm{s}_{6}^{-}$ \\
\hline & & & & $c_{3}^{+}-c_{23}$ & $c_{3}^{-}-c_{21}$ & $\mathrm{E}-\mathrm{C}_{22}$ & $\sigma_{3}-S_{6}^{-}$ & $\sigma_{1}-s_{6}^{+}$ & $\sigma_{2}^{-i}$ \\
\hline & & & & $\sigma_{1}-i$ & $\sigma_{2}-\mathrm{s}_{6}^{-}$ & $\sigma_{3}-\mathrm{s}_{6}^{+}$ & $E-C_{2 I}$ & $c_{3}^{+}-c_{22}$ & $c_{3}^{-}-c_{23}$ \\
\hline & & & & $\sigma_{2}-\mathrm{s}_{6}^{+}$ & $\sigma_{3^{-i}}$ & $\sigma_{1}-s_{6}^{-}$ & $c_{3}^{-}-c_{22}$ & $\mathrm{E}-\mathrm{C}_{23}$ & $c_{3}^{+}-c_{21}$ \\
\hline & & & & $\sigma_{3}-s_{6}^{-}$ & $\sigma_{1}-s_{6}^{+}$ & $\sigma_{2}^{-i}$ & $c_{3}^{+}-c_{23}$ & $c_{3}^{-}-c_{21}$ & $\mathrm{E}-\mathrm{C}_{22}$ \\
\hline
\end{tabular}

$i=\left(\begin{array}{rrr}-1 & 0 & 0 \\ 0 & -1 & 0 \\ 0 & 0 & -1\end{array}\right), S_{6}^{+}=\left(\begin{array}{ccc}1 / 2 & -\gamma & 0 \\ \gamma & 1 / 2 & 0 \\ 0 & 0 & -1\end{array}\right), S_{0}^{-}=\left(\begin{array}{ccr}1 / 2 & \gamma & 0 \\ -\gamma & 1 / 2 & 0 \\ 0 & 0 & -1\end{array}\right)$, $\sigma_{d 1}=\left(\begin{array}{rrr}-1 & 0 & 0 \\ 0 & 1 & 0 \\ 0 & 0 & 1\end{array}\right), \quad \sigma_{d 2}=\left(\begin{array}{ccc}1 / 2 & \gamma & 0 \\ \gamma & -1 / 2 & 0 \\ 0 & 0 & 1\end{array}\right), \sigma_{d 3}=\left(\begin{array}{ccc}1 / 2 & -\gamma & 0 \\ -\gamma & -1 / 2 & 0 \\ 0 & 0 & 1\end{array}\right)$ $C_{21}^{1}=\left(\begin{array}{rrr}1 & 0 & 0 \\ 0 & -1 & 0 \\ 0 & 0 & -1\end{array}\right), \quad C_{22}^{1}=\left(\begin{array}{ccc}-1 / 2 & -\gamma & 0 \\ -\gamma & 1 / 2 & 0 \\ 0 & 0 & -1\end{array}\right), \quad C_{23}^{1}=\left(\begin{array}{ccc}-1 / 2 & \gamma & 0 \\ \gamma & 1 / 2 & 0 \\ 0 & 0 & -1\end{array}\right)$ $C_{3}^{+}=\left(\begin{array}{ccc}-1 / 2 & -\gamma & 0 \\ \gamma & -1 / 2 & 0 \\ 0 & 0 & 1\end{array}\right), \quad C_{3}^{-}=\left(\begin{array}{ccc}-1 / 2 & \gamma & 0 \\ -\gamma & -1 / 2 & 0 \\ 0 & 0 & 1\end{array}\right), \quad E=\left(\begin{array}{lll}1 & 0 & 0 \\ 0 & 1 & 0 \\ 0 & 0 & 1\end{array}\right)$, $\gamma=\frac{(3\}^{1 / 2}}{2}$.

${ }^{1}$ L. D. Landau and E. M. Lifshitz, Statistical Physics, 3rd ed., reviewed by E. M. Lifshitz and L. P. Pitaevskii, translated by J. B. Sykes and M. J. Kearsley (Pergamon, Oxford, 1980).
${ }^{2} J$. L. Birman, in Group Theoretical Methods in Physics, edited by P. Kramer and A. Rieckers (Springer, Berlin, 1978), Vol. 79.

${ }^{3}$ J. D. Barnett, J. Pack, and H. T. Hall, Trans. Am. Crystallog. Assoc. 5, 113 (1969); T. R. Lettieri, E. M. Brody, and W. A. Bassett, Solid State Commun. 26, 235 (1978)

${ }^{4}$ M. V. Jarić, Phys. Rev. Lett. 48, 1641 (1982).

${ }^{5}$ N. F. M. Henry and K. Lonsdale, International Table for X-ray Crystallography (Kynock, Birmingham, 1952).

${ }^{6} \mathbf{L}$. Michel and J. Mozrzymas, Lecture Notes in Physics, edited by $\mathbf{P}$. Kramer and A. Rieckers (Springer, Berlin, 1977), Vol. 79, p. 447.

${ }^{7}$ Joseph L. Birman, Phys. Rev. Lett. 17, 1216 (1966).

${ }^{8} \mathrm{C}$. J. Bradley and A. P. Cracknell, The Mathematical Theory of Symmetry in Solids (Clarendon, Oxford, 1972).

${ }^{9}$ F. E. Goldrich and J. L. Birman, Phys. Rev. 167, 528 (1968); M. V. Jarić and J. L. Birman, Phys. Rev. B 16, 2564 (1977).

${ }^{10}$ D. M. Hatch, Phys. Rev. B 23, 2346 (1981). 